\title{
GMR
}

\section{Quantitative trait loci mapping of the stigma exertion rate and spikelet number per panicle in rice (Oryza sativa L.)}

\author{
M.H. Rahman, P. Yu , Y.X. Zhang, L.P. Sun, W.X. Wu, X.H. Shen, \\ X.D. Zhan, D.B. Chen, L.Y. Cao and S.H. Cheng \\ National Center for Rice Improvement, China National Rice Research Institute, \\ Key Laboratory for Zhejiang Super Rice Research, Hangzhou, Zhejiang, China \\ Corresponding author: S.H. Cheng \\ E-mail: shcheng@mail.hz.zj.cn
}

Genet. Mol. Res. 15 (4): gmr15048432

Received January 13, 2016

Accepted March 21, 2016

Published October 17, 2016

DOI http://dx.doi.org/10.4238/gmr15048432

Copyright $(C 2016$ The Authors. This is an open-access article distributed under the terms of the Creative Commons Attribution ShareAlike (CC BY-SA) 4.0 License.

\begin{abstract}
The stigma exertion rate is a polygenic inherited trait that is important for increased seed yield in hybrid rice breeding. To identify quantitative trait loci (QTL) associated with high stigma exertion rate, we conducted QTL mapping using 134 recombinant inbred lines derived from XieqingzaoB and Zhonghui9308, which have high and low stigma exertion rates, respectively. A total of eight QTLs (qSES6, $q S S E 11, q D S E 1 a, q D S E 1 b, q D S E 10, q D S E 11, q T S E 1$, and qTSE11) for single stigma exertion, double stigma exertion, and total stigma exertion were detected. The locations of qSSE11 and qTSE11 have not been previously reported, and the $q D S E 11$ allele from parent XQZB exhibited a positive additive effect. In addition, three QTLs ( $q S N P 1$, $q S N P 3 a$, and $q S N P 3 b$ ), for spikelet number per panicle were identified. Of note, one QTL ( $q S N P 1$ ) was detected in two different environments (Hainan and Zhejiang). To evaluate the advantage of exerted stigma
\end{abstract}

Genetics and Molecular Research 15 (4): gmr15048432 
for cross-pollination, single, dual, and total stigma exertion should be considered separately for future genetic improvement in the production of rice hybrid seeds. In addition, this study provides information for fine mapping, gene cloning, and marker assisted selection, with emphasis on the latter.

Key words: Rice; Stigma exertion rate; Spikelet number per panicle; QTL mapping

\section{INTRODUCTION}

Rice (Oryza sativa L.) is an important staple cereal food, feeding more than half of the world's population. Increase of rice production is vital to population explosion and global food security (Lou et al., 2014). Hybrid rice that has a yield advantage of $10-20 \%$ over conventional varieties was developed and commercially grown. Together with the application of modern cultivation technology, the rice yield has risen to about 6 tons per hectare. This has contributed to self-sufficiency of food supply in China (Cheng et al., 2007). However, how to improve the efficiency of hybrid seed production remains a major unresolved issue. Stigma exertion rate is a key-contributing factor in hybrid seed production. A high stigma exertion rate is expected to trap more pollen and to improve cross-pollination and the efficiency of hybrid seed production in rice. Exerted stigmas remain viable for approximately 4 days and continue to accept pollination (Long and Shu, 2000; Tian et al., 2004).

Yan et al. (2009) performed association mapping on the stigma exertion rate in a minicore of 90 accessions using 109 DNA markers and found that a simple sequence repeat (SSR) marker RM5, played a major role indicative of high correlation with both dual (DSE) and single stigma exertion (SSE) rates. Using two segregating populations derived from indica cultivar Guangluai-4 and a wild cultivar W1943 (O. rufipogon), Li et al. (2014) demonstrated that the stigma exertion rate is a complex quantitative trait, which is governed by polygenes. Furthermore, those authors performed genetic mapping and validated quantitative trait loci (QTL) associated with stigma exertion rate in rice, and a population of recombinant inbred lines was developed from two superior rice cultivars, Zhongguo Xiangdao (ZX) and Chuanxiang 29B (CX29B), and a total of 11 QTLs for the stigma exertion rate were detected over 2 years; two QTLs on chromosome 1 and four QTLs on chromosome 6 formed two QTL cluster regions, qSe1 and qSe6, respectively. Lou et al. (2014) used the $\mathrm{F}_{2}$ population derived from two indica cytoplasmic male sterility maintainers, Huhan1B and K17B, to map the QTLs that influence stigma exertion in rice. QTLs that influence SSE, DSE, and total stigma exertion (TSE) rates were detected using a linkage map of 92 SSR markers. One, three, and one QTLs were detected for SSE, DSE, and TSE, respectively, on chromosomes 5, 6, and 7.

Zhang et al. (2009) studied a population of recombinant inbred lines (RILs) derived from two indica rice varieties, Zhenshan 97 and HR5, that were used to map QTLs for spikelet number per panicle (SNP). One major QTL ( $q S N P 7)$ and three minor QTLs ( $q S N P 1, q S N P 2$, and $q S N P 3$ ) were identified on chromosomes 7, 1, 2, and 3, respectively. Deshmukh et al. (2010) identified candidate genes for SNP in rice (O. sativa L.) and reported that a large number of well-filled SNPs is an important yield component trait in rice. Additionally, those authors generated a set of RILs derived from a cross between Pusa 1266 (high SNP) and Pusa Basmati 1 (low SNP), and identified four QTLs on chromosomes 3 and 4.

Genetics and Molecular Research 15 (4): gmr15048432 
In the present study, four flowering-related traits, including SSE, DSE, TSE, and SNP, were measured in two different environments (Hainan and Zhejiang) in an RIL population. Here, we identify the QTLs influencing stigma exertion rate and SNP. These results will be useful in facilitating the development of male sterile lines with a high stigma exertion rate and an increase in the SNP, which would be of great value in hybrid rice seed production.

\section{MATERIAL AND METHODS}

\section{Materials and field experiments}

RIL populations consisting of 134 lines were derived from combinations of XieqingzaoB (XQZB) and Zhonghui9308 (ZH9308). XQZB and ZH9308 have high and low stigma exertion rates, respectively. RIL populations and their parents were cultivated at Lingshui, Hainan Island on December 27, 2014 and in Fuyang field of the China National Rice Research Institute, Hangzhou, Zhejiang Province, China on June 18, 2015. Plants were established in six rows, with eight plants per row, and spaces of $30 \times 20 \mathrm{~cm}$ within and between rows, respectively. Standard cultivation practices were followed.

\section{Trait evaluation}

The stigma exertion rate was subdivided into three traits: SSE, DSE, and TSE (Figure 1). At 5-7 days after heading, five normal panicles in each line were sampled from different plants. After the lower side spikelets of the panicle flowered, the panicles were used to determine the stigma exertion rate, as follows.

$$
\begin{aligned}
& \mathrm{SSE}(\%)=[\mathrm{SSE} /(\mathrm{SSE}+\mathrm{DSE}+\text { no stigma exertion })] \times 100 \\
& \mathrm{DSE}(\%)=[\mathrm{DSE} /(\mathrm{SSE}+\mathrm{DSE}+\text { no stigma exertion })] \times 100 \\
& \mathrm{TSE}(\%)=\mathrm{SSE}(\%)+\mathrm{DSE}(\%) \\
& \mathrm{SNP}=\mathrm{SSE}+\mathrm{DSE}+\text { no stigma exertion }
\end{aligned}
$$

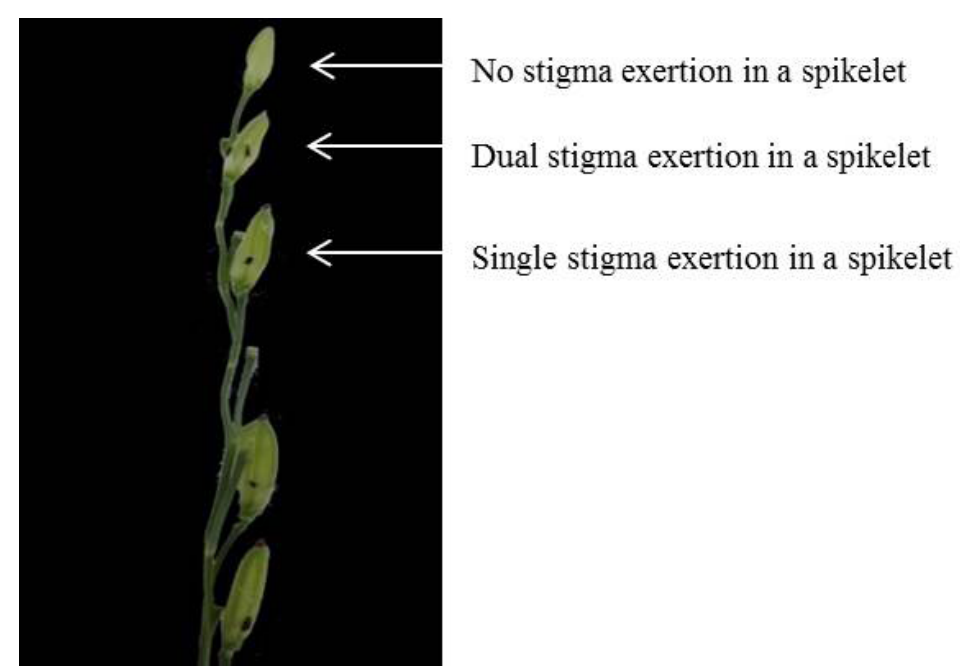

Figure 1. Example of single, dual, and no stigma exertion in a spikelet investigated in this study. 


\section{Construction of a genetic map and QTL analysis}

A total of 198 SSR markers, which were distributed across the rice genome according to previously reported linkage maps, and known polymorphisms between the parents, were used to determine the genotype of the RIL populations (Liang et al., 2012). The genetic linkage map was constructed using the MAPMAKER/Exp version 3.0 program (Lincoln et al., 1992). The entire genome spanned $1814.50 \mathrm{cM}$ with an average interval of $9.16 \mathrm{cM}$ between adjacent markers.

The chromosomal locations of putative QTLs were determined by exclusive composite interval mapping with QTL IciMapping 4.0 (Wang, 2009). A locus with a threshold log of the odds (LOD) value $\geq 2.0$ and $\mathrm{P} \leq 0.05$ was declared as a putative QTL. The contribution rate $\left(\mathrm{H}^{2}\right)$ was estimated as percentage of variance explained by each locus or epistatic pair in proportion to the total phenotypic variance. QTLs were named according to conventions of nomenclature (McCouch, 2008) and in alphabetic order for QTLs on the same chromosome.

\section{RESULTS}

\section{Stigma exertion rate in the RIL and parent populations}

Phenotypic analysis indicated that the SSE, DSE, and TSE rates of the ZH9308 and XQZB parents were higher in plants grown in Zhejiang compared with those grown in Hainan; however, the differences were only statistically significant for the DSE rate in the XQZB parent (Table 1). The SSE, DSE, and TSE in the RIL population exhibited increased values in Zhejiang compared with those in Hainan, and SSE and TSE were significantly different. However, the DSE did not significantly differ between the two locations. The height values for SSE, DSE, and TSE in the RIL population were 52.28, 12.92, and 64.76\% in Hainan and $47.86,22.91$, and $65.95 \%$ in Zhejiang, respectively. SSE (Figure 2A) and TSE (Figure 2C) exhibited continuous variation, which followed a normal distribution in both environments. However, DSE (Figure 2B) deviated significantly from the normal distribution. In addition, transgressive segregation was observed for all traits.

Table 1. Performance of the trait stigma exertion and spikelet number per panicle in the parent and recombinant inbred line (RIL) population.

\begin{tabular}{l|c|c|c|c|c|c|c|c}
\hline \multirow{2}{*}{ Traits } & \multicolumn{2}{|c|}{ ZH9308 \pm SE } & \multicolumn{2}{c|}{ XQZB \pm SE } & \multicolumn{2}{c|}{ RILs (mean) \pm SE } & \multicolumn{2}{c}{ RILs (range) } \\
\cline { 2 - 9 } & Hainan & Zhejiang & Hainan & Zhejiang & Hainan & Zhejiang & Hainan & Zhejiang \\
\hline SSE & $9.36 \pm 0.58$ & $11.07 \pm 0.94$ & $23.99 \pm 3.10$ & $25.59 \pm 2.23$ & $14.99 \pm 0.93^{* *}$ & $18.22 \pm 0.90^{* *}$ & $0.95-52.28$ & $0-47.86$ \\
\hline DSE & $1.19 \pm 0.31$ & $1.35 \pm 0.24$ & $2.03 \pm 0.86^{*}$ & $5.05 \pm 0.56^{*}$ & $3.01 \pm 0.25$ & $3.56 \pm 0.35$ & $0-12.92$ & $0-22.91$ \\
\hline TSE & $10.55 \pm 0.55$ & $12.42 \pm 0.93$ & $26.01 \pm 3.09$ & $30.64 \pm 2.07$ & $18.00 \pm 1.16^{*}$ & $21.71 \pm 1.20^{*}$ & $0.95-64.76$ & $0-65.95$ \\
\hline SNP & $252.20 \pm 7.41$ & $251.20 \pm 6.03$ & $121.40 \pm 3.59^{* * *}$ & $118.80 \pm 2.58^{* * *}$ & $154.59 \pm 3.08^{* * *}$ & $184.46 \pm 3.42^{* * *}$ & $94.60-288.20$ & $91-315.40$ \\
\hline
\end{tabular}

*Significant at the 0.05 level; **significant at the 0.01 level; ***significant at the 0.001 level (analyzed by the Student $t$-test); SSE = single stigma exertion rate; DSE = dual stigma exertion rate; TSE = total stigma exertion rate; $\mathrm{SNP}=$ spikelet number per panicle; $\mathrm{SE}=$ standard error.

\section{SNP in RIL and parent populations}

In both environments, the average SNP was increased in ZH9308 plants compared with XQZB plants (Table 1). SNP of the ZH9308 parent was increased in Hainan compared with Zhejiang; however, the difference was not significant. The XQZB parent had a higher SNP in Hainan than in Zhejiang, and this difference was significant.

Genetics and Molecular Research 15 (4): gmr15048432 
A

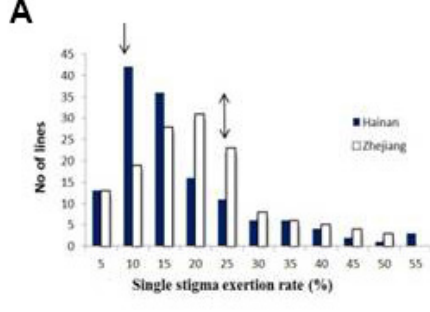

C

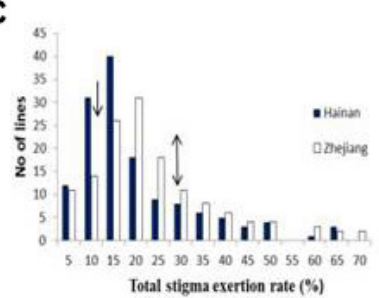

B
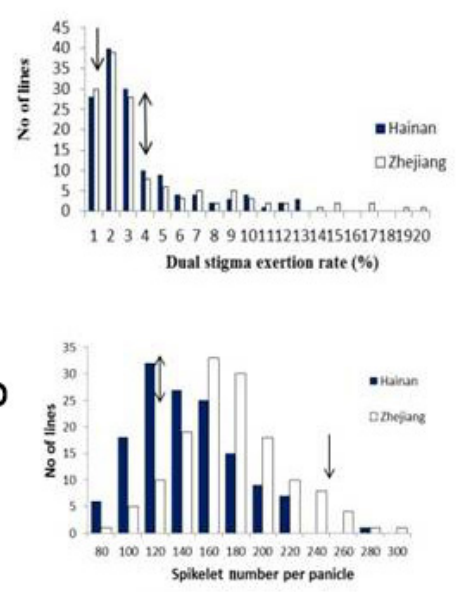

Figure 2. Frequency distribution of A. single exerted stigma (SSE), B. dual exerted stigma (DSE), C. total exerted stigma (TSE), and D. spikelet number per panicle (SNP) in the recombinant inbred line (RIL) population from Hainan and Zhejiang. Single arrows and double-headed arrows indicate the average values from ZH9308 and XQZB parent lines, respectively.

The RIL populations exhibited average SNP values of 154.59 and 184.46 in Hainan and Zhejiang, respectively, and these values significantly differed. SNP ranged from 94.60 to 288.20 and from 91 to 315.40 in Hainan and Zhejiang, respectively. Furthermore, this trait exhibited a normal distribution (Figure 2D).

The SSE, DSE, and TSE traits were significantly correlated with each other in an environmentally dependent manner. No correlation was observed between SNP and stigma exertion. The highest phenotypic correlation was observed between HSSE and HTSE $(r=0.996)$ followed by HDSE and HTSE $(r=0.942)$, and HSSE and HDSE $(r=0.908)$ in Hainan as well as ZJSSE and ZJTSE $(r=0.988)$ and ZJDSE and ZJTSE $(r=0.926)$, and ZJSSE and ZJDSE $(r$ $=0.866$ ) in Zhejiang (Table 2). These results indicated that the lines with higher values for SSE were more likely to exhibit increased values for DSE, which would ultimately increase the TSE.

Table 2. Correlation (Pearson) coefficients among the traits of 134 RIL populations derived from XQZB and ZH9308 parent lines in Hainan and Zhejiang.

\begin{tabular}{l|c|c|c|c|c|c|c|c}
\hline & HSSE & HDSE & HTSE & HSNP & ZJSSE & ZJDSE & ZJTSE & ZJSNP \\
\hline HSSE & 1 & & & & & & \\
\hline HDSE & $0.908^{* *}$ & 1 & & & & & \\
\hline HTSE & $0.996^{* *}$ & $0.942^{* *}$ & 1 & & & & \\
\hline HSNP & 0.073 & 0.109 & 0.083 & 1 & & & & \\
\hline ZJSSE & $0.894^{* *}$ & $0.809^{* *}$ & $0.890^{* *}$ & 0.011 & 1 & & & \\
\hline ZJDSE & $0.801^{* *}$ & $0.781^{* *}$ & $0.809^{* *}$ & 0.138 & $0.866^{* *}$ & 1 & & \\
\hline ZJTSE & $0.891^{* *}$ & $0.827^{* *}$ & $0.891^{* *}$ & 0.039 & $0.988^{* *}$ & $0.926^{* *}$ & 1 & \\
\hline ZJSNP & 0.060 & 0.058 & 0.061 & $0.727^{* *}$ & 0.061 & 0.095 & 0.068 & 1 \\
\hline
\end{tabular}

**Correlation is significant at the 0.01 level (two-tailed); HSSE $=$ Hainan single stigma exertion rate; HDSE = Hainan dual stigma exertion rate; HTSE $=$ Hainan total stigma exertion rate; HSNP $=$ Hainan spikelet number per panicle; ZJSSE = Zhejiang single stigma exertion rate; ZJDSE = Zhejiang dual stigma exertion rate; ZJTSE = Zhejiang total stigma exertion rate; ZJSNP $=$ Zhejiang spikelet number per panicle. 


\section{QTL analysis of stigma exertion rate}

Eight QTLs for stigma exertion rate were identified with LOD values varying from 2.04 to 2.68 and were distributed on chromosomes $1,6,10$, and 11 in both environments (Table 3 and Figure 3). The phenotypic variance explained by each QTL for stigma exertion rates ranged from 6.49 to $8.90 \%$. For the SSE rates, two QTLs, qSSE6 and qSSE11, were detected on chromosomes 6 and 11, respectively, QTL qSSE6 was identified in Hainan, and $q S S E 11$ was identified in Zhejiang. These QTLs explained $7.47 \%$ of the phenotypic variation in Hainan and $8.37 \%$ of the phenotypic variation in Zhejiang, and the alleles were derived from the XQZB parent line. The additive effect was 3.05 and 3.18, respectively. For the DSE rates, four QTLs ( $q D E S 1 a, q D S E 1 b, q D S E 10$, and qDSE11) were detected on chromosomes 1, 1, 10, and 11, respectively. The QTL qDSE10 was identified in Hainan and explained $7.59 \%$ of the phenotypic variation, whereas others found in Zhejiang explained 7.92, 8.90, and $7.28 \%$ of the phenotypic variation. The alleles $q D E S 1 a, q D S E 1 b$, and $q D S E 10$ were inherited from the ZH9308 parent line with negative effects of $1.17,1.27$, and 0.81 , respectively. Conversely, the $q D S E 11$ allele from the XQZB parent line exhibited a positive additive effect of 1.16. Regarding the total stigma exertion rates, two QTLs were detected on chromosome 1 and 11, respectively. The QTLs qTSE 1 and qTSE11 on chromosomes 1 and 11, respectively, explained 6.49 and $8.03 \%$ of the phenotypic variation in Zhejiang. The qTSE1 and qTSE11 alleles were derived from the ZH9308 and XQZB parents, respectively. The former had a negative effect of 3.73, whereas the latter had a positive additive effect of 4.18 .

Table 3. Putative quantitative trait loci (QTLs) for stigma exertion rate and spikelet number per panicle detected in an RIL population derived from the XQZB and ZH9308 parent lines in Hainan and Zhejiang.

\begin{tabular}{|c|c|c|c|c|c|c|c|c|c|c|c|}
\hline \multirow[t]{2}{*}{ Trait } & \multirow[t]{2}{*}{ QTL } & \multirow[t]{2}{*}{$\mathrm{Chr}$} & \multirow[t]{2}{*}{ Interval } & \multirow[t]{2}{*}{ Distance $(\mathrm{cM})$} & \multirow[t]{2}{*}{ Sources } & \multicolumn{3}{|c|}{ Hainan-2014-15 } & \multicolumn{3}{|c|}{ Zhejiang-2015 } \\
\hline & & & & & & LOD & $\mathrm{A}$ & PVE (\%) & LOD & $\mathrm{A}$ & PVE (\%) \\
\hline \multirow[t]{2}{*}{ SSE } & qSSE6 & 6 & RM587- RM510 & 14.00 & XQZB & 2.04 & 3.05 & 7.47 & & & \\
\hline & qSSE11 & 11 & RM5704-RM4469 & 34.00 & XQZB & & & & 2.48 & 3.18 & 8.37 \\
\hline \multirow[t]{4}{*}{$\overline{D S E}$} & qDSE1a & 1 & RM7278-RM6324 & 16.00 & ZH9308 & & & & 2.22 & -1.17 & 7.92 \\
\hline & qDSE1b & 1 & RM1-RM3746 & 34.00 & ZH9308 & & & & 2.68 & -1.27 & 8.90 \\
\hline & qDSE10 & 10 & RM474-RM6646 & 23.00 & ZH9308 & 2.19 & -0.81 & 7.59 & & & \\
\hline & qDSE11 & 11 & RM167-RM5704 & 33.00 & XQZB & & & & 2.14 & 1.16 & 7.28 \\
\hline \multirow[t]{2}{*}{ TSE } & qTSE1 & 1 & RM1-RM3746 & 34.00 & ZH9308 & & & & 2.10 & -3.73 & 6.49 \\
\hline & qTSE11 & 11 & RM5704-RM4469 & 34.00 & XQZB & & & & 2.38 & 4.18 & 8.03 \\
\hline \multirow[t]{3}{*}{ SNP } & qSNP1 & 1 & RM1- RM3746 & 52.00 & ZH9308 & 3.70 & -12.17 & 11.21 & 3.33 & -12.88 & 10.19 \\
\hline & qSNP3a & 3 & RM168-RM143 & 133.00 & ZH9308 & 3.99 & -18.22 & 25.04 & & & \\
\hline & qSNP3b & 3 & RM282-RM6283 & 42.00 & ZH9308 & & & & 3.79 & -17.39 & 17.38 \\
\hline
\end{tabular}

$\mathrm{A}=$ additive effect of each QTL; PVE = phenotypic variance explained by each QTL; LOD = logarithm of odds; $q S S E=\mathrm{QTL}$ for single stigma exertion rate; $q D S E=\mathrm{QTL}$ for double stigma exertion rate; $q T S E=\mathrm{QTL}$ for total stigma exertion rate; $q S N P=\mathrm{QTL}$ for spikelet number per panicle.

\section{QTL analysis of SNP}

Three QTLs for SNP with LOD values varying from 3.33 to 3.99 were detected on chromosomes 1 and 3 in both environments (Table 3 and Figure 3). The phenotypic variance explained by each QTL ranged from 10.19 to $25.04 \%$. The QTL located on chromosome 1 was flanked by RM1-RM3746, whereas the QTL on chromosome 3 was flanked by RM168RM143 and RM282-RM6283. qSNP1 was identified in both environments and explained 
11.21 and $10.19 \%$ of the phenotypic variation in Hainan and Zhejiang, respectively. $q S N P 3 a$ was identified in Hainan with $25.04 \%$ of the phenotypic variation, and $q S N P 3 b$ was identified in Zhejiang with $17.38 \%$ of the phenotypic variation. The alleles of these QTLs were derived from the ZH9308 parent, and all exhibited explained negative effects (Table 3).

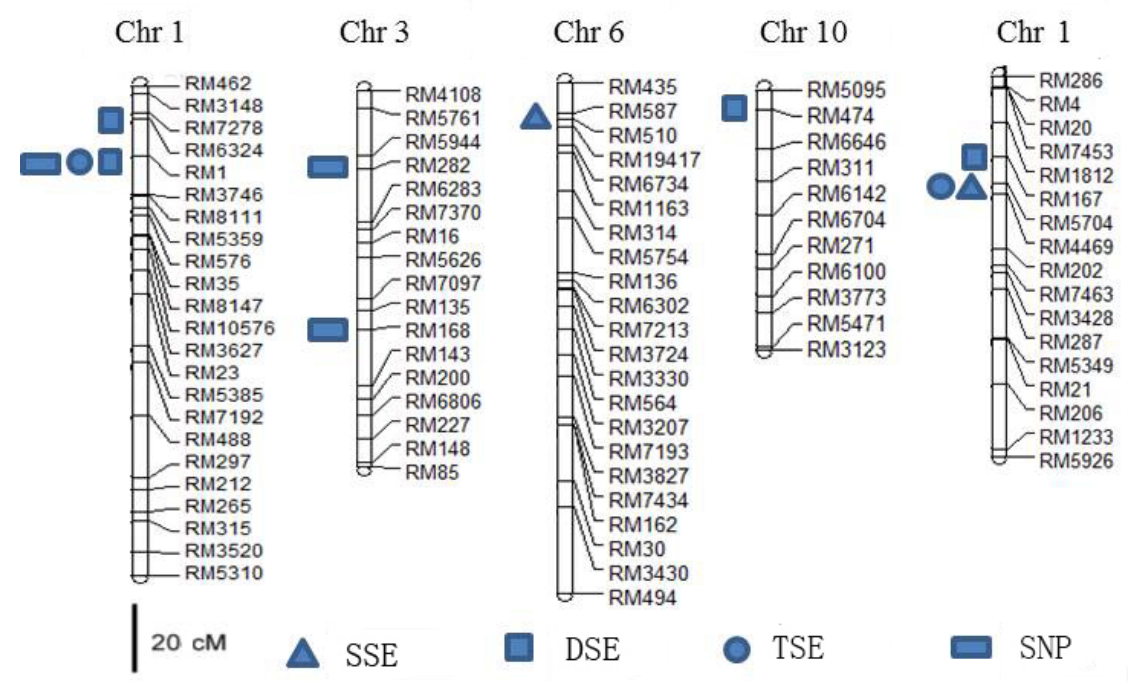

Figure 3. Chromosomal locations of QTL for the percentage of SSE, DSE, TSE, and SNP in RIL populations from the cross between XQZB and ZH9308. Triangle, square, circle, and rectangle represent putative regions of QTLs for SSE, DSE, TSE, and SNP, respectively.

Data were collected in March, April, and May 2015, in Hainan and in August, September, and October 2015 in Zhejiang. The temperatures of the two environments in Hainan and Zhejiang during the data collection periods of March-August, April-September, and May-October were significantly different (Table 4).

\section{Table 4. Comparative study of monthly temperatures in two environments in Hainan and Zhejiang.}

\begin{tabular}{l|c|c|l|c|c}
\hline \multicolumn{3}{|c|}{ Hainan temperature $\left({ }^{\circ} \mathrm{C}\right), 2015$} & \multicolumn{3}{c}{ Zhejiang temperature $\left({ }^{\circ} \mathrm{C}\right), 2015$} \\
\hline Month & Average $\pm \mathrm{SE}$ & Ranges & Month & Average $\pm \mathrm{SE}$ & Ranges \\
\hline March & $25.26 \pm 0.17^{* *}$ & $24.00-27.50$ & August & $27.89 \pm 0.42^{* * * *}$ & $24.00-33.00$ \\
\hline April & $26.42 \pm 0.35^{* * *}$ & $21.50-28.50$ & September & $23.78 \pm 0.34^{* * *}$ & $20.00-27.50$ \\
\hline May & $30.00 \pm 0.14^{* * *}$ & $29.00-31.50$ & October & $19.39 \pm 0.38^{* * *}$ & $13.00-23.50$ \\
\hline
\end{tabular}

**Significant at the 0.01 level. ***Significant at the 0.001 level (analyzed by the Student $t$-test) SE $=$ standard error.

Analysis of variance (ANOVA) based on a fixed-effect model revealed highly significant variance between the two environments (Hainan and Zhejiang) in the RIL population (Table 5). Increased values were observed in Zhejiang compared with Hainan for all traits except for SNP (Table 1). Clearly, environmental factors in Zhejiang favored the stigma exertion rate. 
Table 5. Results of ANOVA based on a fixed-effect model of the 134 RIL populations in two environments.

\begin{tabular}{|c|c|c|c|c|c|}
\hline Trait & Sources of variation & d.f. & MS & F-value & $P$ value \\
\hline \multirow[t]{3}{*}{ SSE } & Line & 133 & 22.47 & $17.80^{* * *}$ & $2 \mathrm{E}-48$ \\
\hline & Environments & 1 & 701.49 & $56.15^{* * *}$ & $8.39 \mathrm{E}-12$ \\
\hline & Residual & 133 & 12.50 & & \\
\hline \multirow[t]{3}{*}{ DSE } & Line & 133 & 22.32 & $6.63 * * *$ & $8.94 \mathrm{E}-25$ \\
\hline & Environments & 1 & 20.28 & $6.02 * *$ & 0.015 \\
\hline & Residual & 133 & 3.36 & & \\
\hline \multirow[t]{3}{*}{ TSE } & Line & 133 & 371.31 & $17.28^{* * *}$ & $1.16 \mathrm{E}-47$ \\
\hline & Environments & 1 & 920.39 & $42.85 * * *$ & $1.17 \mathrm{E}-09$ \\
\hline & Residual & 133 & 21.47 & & \\
\hline \multirow[t]{3}{*}{ SNP } & Line & 133 & 2553.39 & $6.22 * * *$ & $2.01 \mathrm{E} 23$ \\
\hline & Environments & 1 & 59779.13 & $145.70^{* * *}$ & $4.08 \mathrm{E} 23$ \\
\hline & Residual & 133 & 410.27 & & \\
\hline
\end{tabular}

**Significant at the 0.01 level; ***significant at the 0.001 level; Line, the RIL population; Environments, Hainan and Zhejiang; MS, means square.

\section{DISCUSSION}

Stigma exertion rate is a female parental trait important for improving hybrid seed production in rice. In this study, we report QTL for stigma exertion rate that were detected in two different environments. The exerted stigma is fragile and can be easily damaged by environmental conditions such as wind, water stress, and physical interruption, during the flowering period (Yan et al., 2009). In this study, we made several improvements to previously used methods when measuring the stigma exertion rate. In some previous studies, panicles were collected at flowering to avoid damage to exerted stigmas and to improve the accuracy of the collected data (Uga et al., 2003; Yan at al., 2009), whereas in other studies, panicles were collected at approximately 7-10 days post-flowering (Miyata et al., 2007; Takano-kai et al., 2011). Although some exerted stigmas are vulnerable, we noted that the pistil style in the flower remains discernible until the grain-filling period. In the present study, we sampled five main panicles from the parent and five from each line of the RIL population at 5-7 days after heading, when the lower spikelets of the panicle had flowered. The stigma exertion rate is affected by temperature. According to our observations, a temperature of $18^{\circ}-25^{\circ} \mathrm{C}$ and conditions of high light intensity are favorable for stigma exertion. The temperatures of the two environments in Hainan and Zhejiang significantly differed during the flowering period (Table 4). ANOVA, based on a fixed-effect model, revealed highly significant variance between the RIL populations in the two environments (Table 5). Increased values were observed for all traits in Zhejiang compared with Hainan, with the exception of SNP (Table 1). These data clearly demonstrate that the environmental factors in Zhejiang favored a high stigma exertion rate. The identified QTLs that were related to the stigma exertion rate were not stable in both environments due to different environmental factors, such as temperature, wind, water stress, and physical interruption; whereas the QTL related to SNP (qSNP1) was stable in both environments.

In this study, a total of eight QTLs for stigma exertion rate were detected in both environments (Hainan and Zhejiang). We identified two markers for stigma exertion on chromosome 1 (RM7278 and RM1) that were associated with qDSE1a, qDSE1b, and qTSE1; one marker on chromosome 6 (RM587) that was associated with qSSE6; one marker on chromosome 10 (RM474) that was associated with qDSE10; and two markers on chromosome 11 (RM5704 and RM167) that were associated with qSSE11, qDSE11, and qTSE11. Numerous

Genetics and Molecular Research 15 (4): gmr15048432 
studies have described QTLs associated with SSE, DSE, and TSE in rice. Specifically, five QTLs were found to be distributed on chromosomes 2 and 3 (Li et al., 2003); nine QTLs were found on chromosomes 1, 2, 5, and 8 (Yu et al., 2006); fifteen on chromosomes 1, 5, 6, 7, 8, 9, 10, and 11 (Yan et al., 2009); six on chromosomes 1, 2, 5, and 8 (Songpig et al., 2009); 11 on chromosomes 1, 3, 6, 7, 9, 10, and 12 (Li et al., 2014); and five on chromosomes 5, 6, and 7 (Lou et al., 2014). In this study, we demonstrated that the QTL $q D S E 1 a$ (distance $16.0 \mathrm{cM}$ ) between SSR markers RM7278 and RM6324 on chromosome 1 is related to DSE (Figure 3). Li et al. (2014) also identified one QTL that controls DSE in rice, $q D S E-1$ (distance $15.5 \mathrm{cM}$ ), close to the SSR marker RM10105 on the same chromosome and in the same region. The chromosome regions harboring $q D S E-1$ and $q D S E 1 a$ overlap, suggesting that these two QTLs may be identical. This finding confirms the accuracy of the stable QTLs identified in the present study. We identified QTL qSSE6 (distance $14.0 \mathrm{cM}$ ), which is related to SSE and is located between SSR markers RM587 and RM510. Yan et al. (2009) identified one QTL controlling SSE in rice, $q S S E-6$ (distance $0.5 \mathrm{cM}$ ), which is located $13.5 \mathrm{cM}$ from the SSR marker RM133 on chromosome 6. In the present study, we identified QTL qDSE10 (distance 23.0), which is related to DSE and is located between SSR markers RM474 and RM6646. Li et al. (2014) identified one QTL controlling DSE in rice, $q D S E-10$ (distance $40.0 \mathrm{cM}$ ), which is close to the SSR marker RM271 located $17 \mathrm{cM}$ away on chromosome 10. We identified the QTL qDSE11 (distance $33.0 \mathrm{cM}$ ), which is related to DSE and is located between SSR markers RM167 and RM5704. Yan et al. (2009) also identified one QTL controlling DSE in rice, qSSE-11 (distance $8.7 \mathrm{cM}$ ), which is close to the SSR marker RM7203 located $24.7 \mathrm{cM}$ away on chromosome 11. To our knowledge, the qSSE11 and qTSE11 regions have not been previously reported.

In this study, we detected a total of three QTLs for SNP in two environments (Hainan and Zhejiang) on chromosomes 1 and 3, which are related to SSR markers (RM1, RM168, and RM282). The QTLs $q S N P 1, q S N P 3 a$, and $q S N P 3 b$ were associated with the three markers. Markers associated with SNP have previously been reported. Six QTLs are distributed on chromosomes 2, 3, 4, 5, 9, and 11 (Yu et al., 2006; Songpig et al., 2009); four QTLs are distributed on chromosomes 1, 2, 3, and 7 (Zhang et al., 2009); four on chromosomes 3 and 4 (Deshmukh et al., 2010); three on chromosomes 1, 2, and 7 (He et al., 2010); and two on chromosomes 3 and 6 (Liang et al., 2012).

In the present study, one QTL related to SNP (qSNP1) was identified between SSR markers RM1 and RM3746 on chromosome 1. Zhang et al. (2009) and He et al. (2010) also reported one QTL related to SNP (qSNP-1) on the same chromosome. We identified two QTLs related to SNP (qSNP3a and qSNP3b) on chromosome 3. Previous studies (Yu et al., 2006; Songpig et al., 2009; Deshmukh et al., 2010; Liang et al., 2012) have also reported two QTLs related to SNP on the same chromosome.

For the commercial use of hybrid rice cultivation as hybrid rice seed production, maternal lines must have exerted stigma that trap more pollen dispersed from the male parent, thus overcoming the barrier of pollination and increasing the yield of hybrid seeds. QTLs with large effects have been identified as valuable resources for the genetic improvement of quantitative traits (Jiang et al., 2004; Toojinda et al., 2005). In this study, the interval flanked by the SSR markers RM5704 and RM4469 on chromosome 11 had an important effect. If qSSE11 and DSE11 were two tightly linked QTLs, then the combination between these loci could improve both SSE and DSE. Otherwise, DSE is a more favored parameter than SSE, due to the exertion of dual stigmas, which doubles the probability of outcrossing for the production of hybrid seed compared with the exertion of one out of two stigmas in rice. Therefore, the

Genetics and Molecular Research 15 (4): gmr15048432 
application of $q D S E 11$ to improve DSE could be selected by marker-assisted selection (MAS) in rice breeding. A significant positive correlation was observed among SSE, DSE, and TSE.

In summary, we identified six SSR markers for stigma exertion distributed on chromosomes $1,6,10$, and 11; five markers in different regions identified in previous studies on chromosomes $6,10,11$, and one marker on chromosome 1 in a similar region to that identified in previous studies. In addition, we identified three markers for SNP that were distributed on chromosomes 1 and 3, consistent with previous reports. The addition of new regions increases the descriptive power of QTLs associated with stigma exertion and SNP. The SSE rate is sufficient for hybrid rice seed production. To evaluate the advantage of exerted stigma for cross-pollination, SSE, DSE, and TSE should be considered separately for future genetic improvement for the high production of rice hybrid seeds; this study also provides information for fine mapping, gene cloning, and MAS, with emphasis on the latter.

\section{Conflicts of interest}

The authors declare no conflict of interest.

\section{ACKNOWLEDGMENTS}

Research supported by the Natural Science Foundation of China (\#31101203), the National Key Transform Program (\#2014ZX08001-002), the National Natural Science Foundation of China (\#31501290), the Zhejiang Provincial Natural Science Foundation of China (Grant \#LQ14C130003), and the Super Rice Breeding Innovation Team and Rice Heterosis Mechanism Research Innovation Team of the Chinese Academy of Agricultural Sciences Innovation Project (CAAS-ASTIP-2013-CNRRI).

\section{REFERENCES}

Cheng SH, Zhuang JY, Fan YY, Du JH, et al. (2007). Progress in research and development on hybrid rice: a superdomesticate in China. Ann. Bot. 100: 959-966. http://dx.doi.org/10.1093/aob/mcm121

Deshmukh R, Singh A, Jain N, Anand S, et al. (2010). Identification of candidate genes for grain number in rice (Oryza sativa L.). Funct. Integr. Genomics 10: 339-347.http://dx.doi.org/10.1007/s10142-010-0167-2

He Q, Zhang K, Xu C and Xing Y (2010). Additive and additive $\times$ additive interaction make important contributions to spikelets per panicle in rice near isogenic (Oryza sativa L.) lines. J. Genet. Genomics 37: 795-803. http://dx.doi. org/10.1016/S1673-8527(09)60097-7

Jiang GH, Xu CG, Tu JM, Li XH, et al. (2004). Pyramiding of insect-and disease-resistance genes into an elite indica, cytoplasm male sterile restorer line of rice, 'Minghui 63'. Plant Breed. 123: 112-116. http://dx.doi.org/10.1046/ j.1439-0523.2003.00917.X

Li P, Feng F, Zhang Q, Chao Y, et al. (2014). Genetic mapping and validation of quantitative trait loci for stigma exertion rate in rice. Mol. Breed. 34: 2131-2138. http://dx.doi.org/10.1007/s11032-014-0168-2

Li WH, Dong GJ, Hu XM, Teng S, et al. (2003). QTL analysis for percentage of exserted stigma in rice (Oryza sativa L.). Yi Chuan Xue Bao 30: 637-640.

Liang Y, Zhan X, Gao Z, Lin Z, et al. (2012). Mapping of QTLs association with important agronomic traits using three populations derived from super hybrid rice Xieyou9308. Euphytica 184: 1-13. http://dx.doi.org/10.1007/s10681$\underline{011-0456-4}$

Lincoln SE, Daly MJ and Lander E (1992). Constructing Genetic Map with Mapmaker/ Exp 3:0 Whitehead Institute Technical report. 3rd edn. Whitehead Institute, Cambridge.

Long LH and Shu K (2000). Increasing outcrossing rate of indica hybrid rice. J. Hunan Agric. Univ. 26: 205-208.

Lou J, Yue GH, Yang WQ, Mei HW, et al. (2014). Mapping QTLs influencing stigma exertion in rice. Bulg. J. Agric. Sci. 20: $1450-1456$.

Genetics and Molecular Research 15 (4): gmr15048432 
McCouch SR (2008). Gene nomenclature system for rice. Rice 1: 72-84. http://dx.doi.org/10.1007/s12284-008-9004-9

Miyata M, Yamamoto T, Komori T and Nitta N (2007). Marker-assisted selection and evaluation of the QTL for stigma exsertion under japonica rice genetic background. Theor. Appl. Genet. 114: 539-548. http://dx.doi.org/10.1007/ s00122-006-0454-4

Songpig H, Ying Z, Lin Z, Xudong Z, et al. (2009). QTL analysis of floral traits of rice (Oryza sativa L.) under wellwatered and drought stress conditions. Genes Genomics 31: 173-181. http://dx.doi.org/10.1007/BF03191150

Takano-kai N, Doi K and Yoshimura A (2011). GS3 participates in stigma exertion as well as seed length in rice. Breed. Sci. 61: 244-250. http://dx.doi.org/10.1270/jsbbs.61.244

Tian DC, Huang SK, Duan YG and Wang YH (2004). The relationship between flowering and pollination time and outcrossing rate of male sterile lines in hybrid rice seed production. Hybrid Rice 19: 50-54.

Toojinda T, Tragoonrung S, Vanavichit A, Siangliw JL, et al. (2005). Molecular breeding for rainfed lowland rice in the Mekong region. Plant Prod. Sci. 8: 330-333. http://dx.doi.org/10.1626/pps.8.330

Uga Y, Fukuta Y, Cai HW, Iwata H, et al. (2003). Mapping QTLs influencing rice floral morphology using recombinant inbred lines derived from a cross between Oryza sativa L. and Oryza rufipogon Griff. Theor. Appl. Genet. 107: 218226. http://dx.doi.org/10.1007/s00122-003-1227-y

Wang J (2009). Inclusive composite interval mapping of quantitative trait genes. Acta Agron. Sin. 35: 239-245. http:// dx.doi.org/10.3724/SP.J.1006.2009.00239

Yan WG, Li Y, Agrama HA, Luo D, et al. (2009). Association mapping of stigma and spikelet characteristics in rice (Oryza sativa L.). Mol. Breed. 24: 277-292. http://dx.doi.org/10.1007/s11032-009-9290-y

Yu XQ, Mei HW, Luo LJ, Liu GL, et al. (2006). Dissection of additive, epistatic effect and Q x E interaction of quantitative trait loci influencing stigma exsertion under water stress in rice. Yi Chuan Xue Bao 33: 542-550. http://dx.doi. org $/ 10.1016 / \mathrm{S} 0379-4172(06) 60083-8$

Zhang Y, Luo L, Liu T, Xu C, et al. (2009). Four rice QTL controlling number of spikelets per panicle expressed the characteristics of single Mendelian gene in near isogenic backgrounds. Theor. Appl. Genet. 118: 1035-1044. http:// dx.doi.org/10.1007/s00122-008-0960-7

Genetics and Molecular Research 15 (4): gmr15048432 